\title{
8. A BRIEF SURVEY OF UPPER-AIR SPECTRA
}

\author{
P. M. Millman \\ (National Research Council, Ottawa, Canada, and \\ Consultant, Avco Everett Research Laboratory, U.S.A.)
}

\begin{abstract}
A BST RACT
The atomic multiplets and molecular band systems identified in six categories of upper-air sources are studied. These total 510 multiplets of 38 atoms and ions, and 54 band systems of 27 molecules. The average excitation decreases as one progresses from the sources resulting from the influx of the faster and smaller particles to those produced by the slower and larger objects.
\end{abstract}

Our upper atmosphere is continually bombarded by a variety of particles from space, ranging all the way from electrons, protons and various atoms, up through interplanetary dust to larger meteoritic material. The more massive examples of the latter fall to the Earth as meteorites. During the last decade, man-made objects have been added to the natural influx of solid matter into the Earth's atmosphere.

Most of the material noted above has a high velocity relative to the Earth, and produces visible luminosity of various types on entering the upper atmosphere. It is proposed to summarize here the major characteristics of the light from these upperair sources, and to indicate the inter-relations among the various categories of spectra.

For convenience the observational material will be considered under six main headings: (1) aurora, (2) airglow, (3) meteors, (4) meteor trains, (5) rocket bodies, and (6) re-entry vehicles.

For the first two categories data have been taken from recent publications in the field, in particular, work by Bates (1960) and Chamberlain (1961). For material on meteors and meteor trains, the writer has extended his own previous surveys (Millman, 1963) to include the more recent observations now available. For the last 5 years the writer has been a part-time consultant at the Avco Everett Research Laboratory. The work here has involved the study of re-entry spectra, photographed as part of an extensive airborne coverage by this Laboratory of rocket and vehicle re-entries on both the Atlantic and Pacific ranges. In the data summarized, the writer has combined his own original identifications with the work of a number of experimenters in the re-entry data group of Avco Everett. However, the final selection of what has been included has been made by the writer.

A major portion of the auroral luminosity, and a minor fraction of airglow luminosity, owe their origin to the arrival of high-energy electrons from the Sun. Also

Kresák and Millman (eds.), Physics and Dynamics of Meteors, 84 90. C. I.A.U. 
effective are solar protons, neutral atoms, and ultraviolet radiation. The velocities of the protons are measured in thousands of kilometers a second, while those of the electrons in tens of thousands of kilometers a second. Visible meteors result from the entry of solid particles with masses ranging from a small fraction of a gram up to many kilograms. Most of the spectroscopic data for meteors and their trains refer to objects with original masses in the 1-10000 gram range, which enter the atmosphere at speeds of $15-70 \mathrm{~km} / \mathrm{sec}$. Rocket bodies have masses of a few thousand kilograms and velocities in the range $2-7 \mathrm{~km} / \mathrm{sec}$. Re-entry vehicles have similar velocities and masses somewhat less than the rocket bodies (project FIRE achieved a reentry speed of about $11 \mathrm{~km} / \mathrm{sec}$ ).

It is instructive to note the height distribution of these sources. Figure 1 shows the

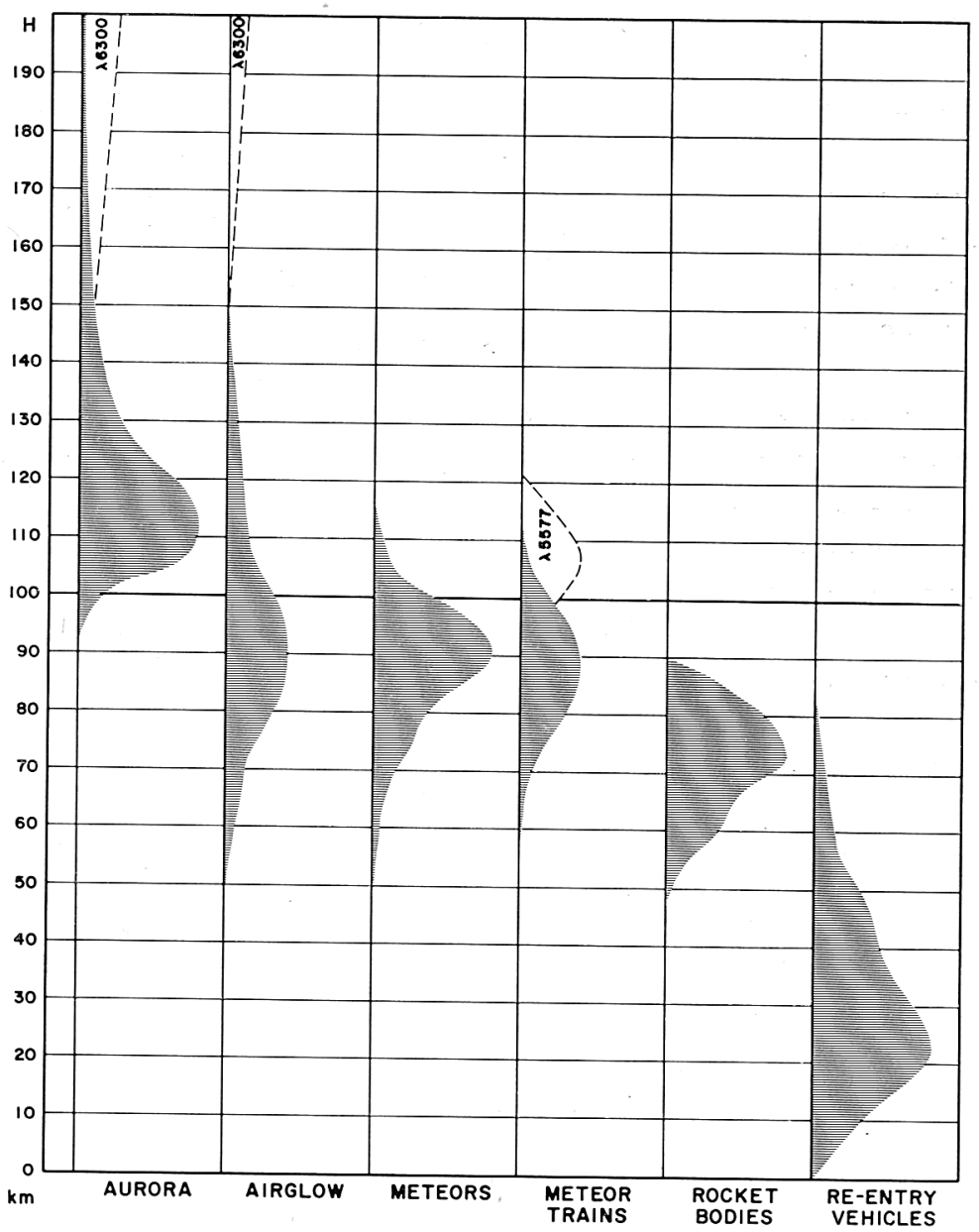

FIG. 1. A qualitative plot of the relative heights of six categories of upper-air sources. 
relative heights for the six categories of data presented here. In the case of the forbidden lines of neutral oxygen, the average height distribution may deviate widely from the rest of the data and hence has been plotted separately for the red lines in the case of the aurora and airglow, and for the green line in the case of meteor trains. In general, as we move from the smaller and faster particles to the larger and slower objects, the heights decrease.

Typical examples from the photographic data available are reproduced in Figures 2-6. Multiplet numbers are from the table by Moore (1945). In identifying band systems and band heads, primary references have been Gatterer et al. (1957), and Pearse and Gaydon (1963). Multiplets and band systems that cannot be independently identified in the photographs have not been included in this study, even though one can often conclude from analogy that they are contributing to the luminosity observed. In the analysis of poor-quality spectra, identifications have been made on the basis of a knowledge of high-quality spectra of the same type.

In all 429 multiplets of 28 neutral atoms, 81 multiplets of 10 singly ionized atoms, 51 band systems of 25 neutral molecules, and 3 band systems of 2 ionized molecules have been identified with reasonable certainty. In general this covers the observational material reduced up to the end of 1966, with a few additions from the first half of 1967.

Upper-air spectra are primarily low-excitation phenomena. Frequently the transitions arising from the ground state of the atom or molecule are relatively strong, even when compared with laboratory spectra of the lowest excitation. Except for oxygen and nitrogen, the ionization potentials of the ions identified lie in the range 5.2-8.1 volts. In Table 1 are listed the mean excitations for all the multiplets of the neutral atoms, except for the forbidden lines. As would be expected the dominant features are of lower excitation than the others, and as we move from the smaller, faster particles to the larger, slower objects we move to lower average excitations. Another evidence of this trend is the much larger number of molecules identified in the re-entry objects as compared with meteors. Apart from the various molecules of

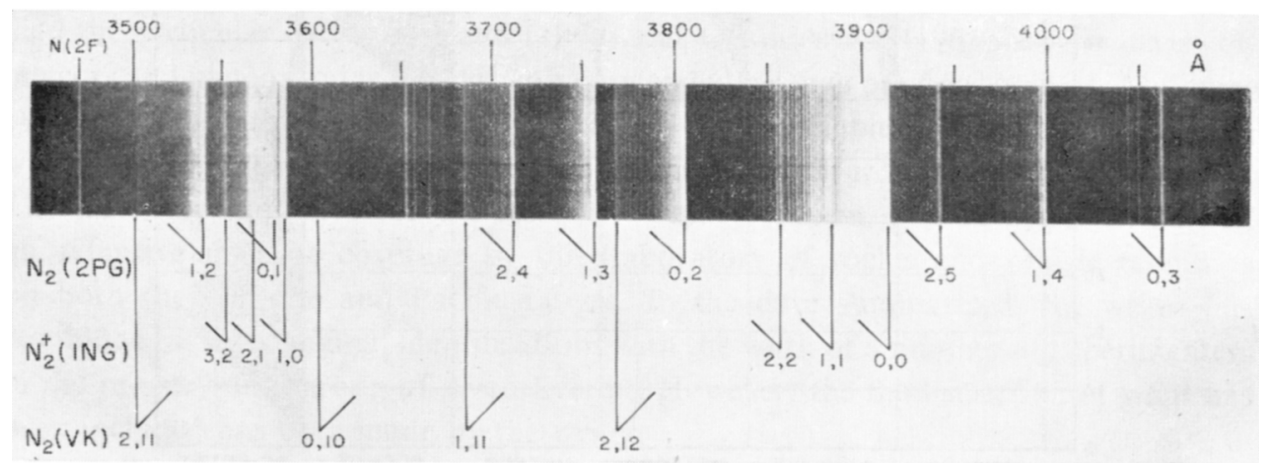

FIG. 2. Portion of the auroral spectrum, photographed at the University of Saskatchewan, Saskatoon. Canada. (Courtesy A. Vallance Jones.) 

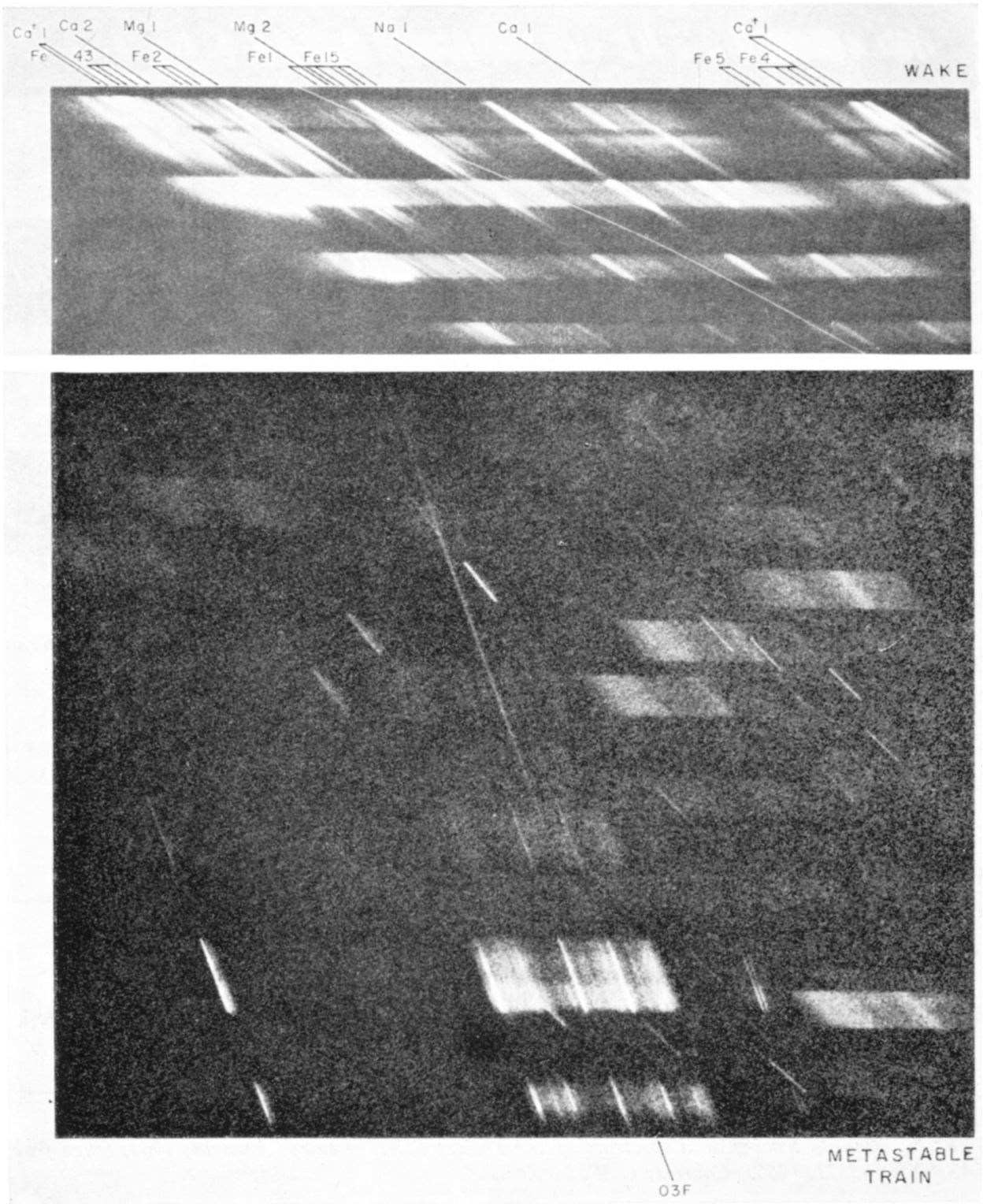

Fig. 3. Typical meteor spectra, photographed at the Springhill Meteor Observatory, showing: (above) the low-level multiplets of various neutral atoms enhanced in the meteor wake, (below) a forbidden oxygen line in the metastable train. 


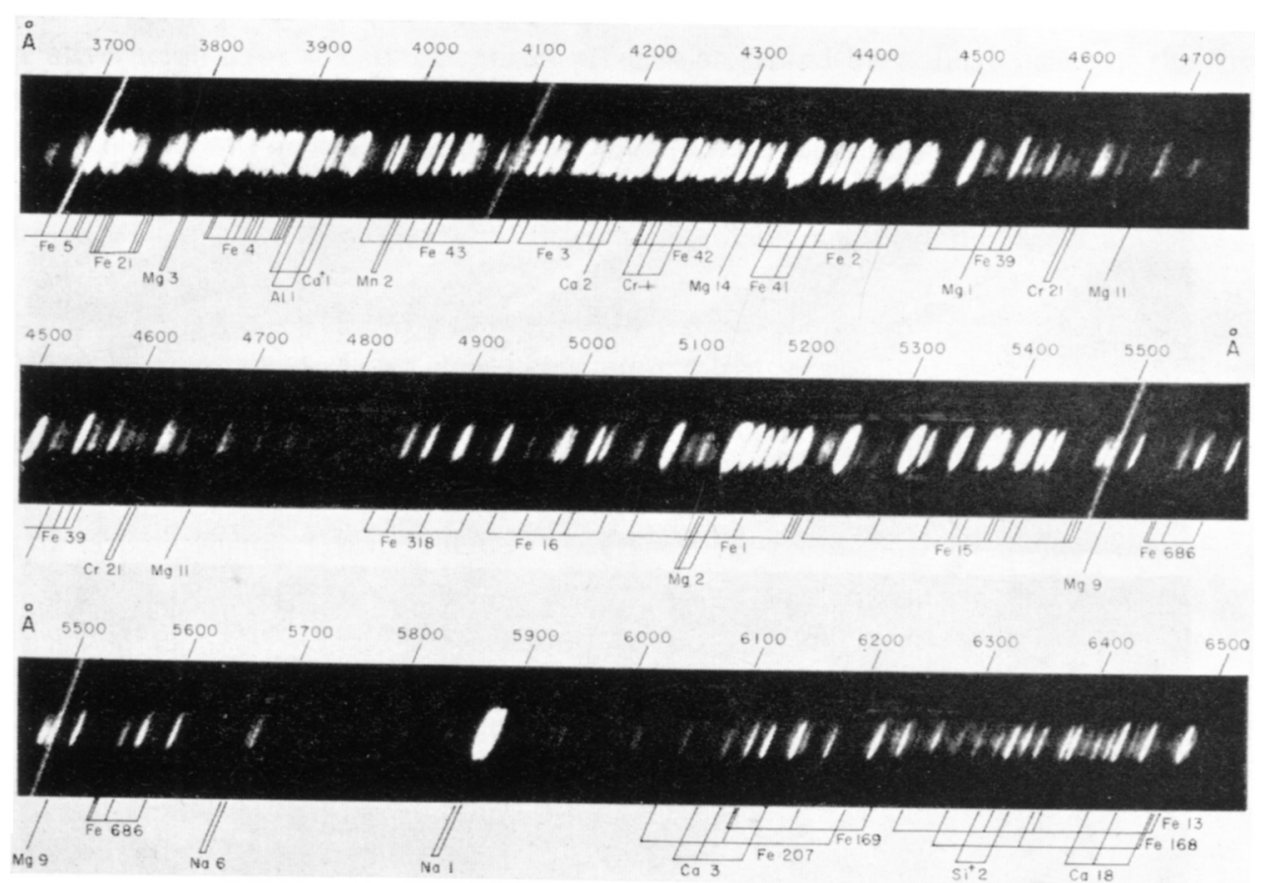

FIG. 4. A high-dispersion meteor spectrum with many iron lines, photographed at the Ondřejov Observatory, Czechoslovakia. (Courtesy Z. Ceplecha.)

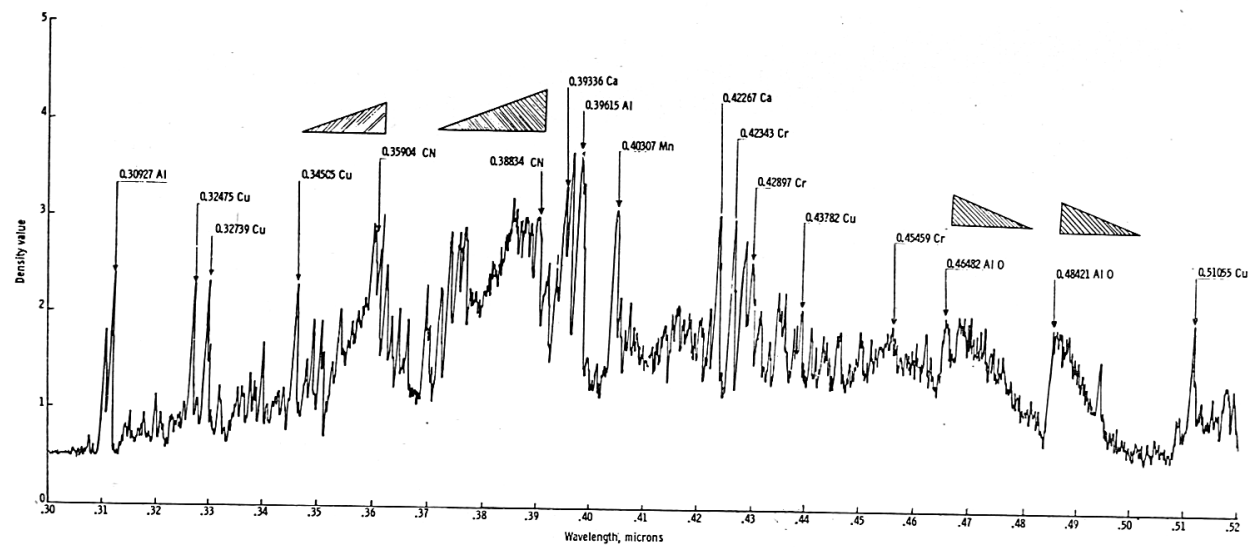

FIG. 5. Density tracing of a spectrum of the Project FIRE re-entry, April 14, 1964, taken from NASA-TN D-3571, 1966. (Courtesy C.W. McKee.)

nitrogen and oxygen, which may have relatively high excitations, the remainder of the molecules identified average 2.9 volts as the upper level for those seen in rocket bodies, and 2.6 volts for the vehicles.

In the case of a large majority of upper-air spectra, it is difficult to judge the com- 

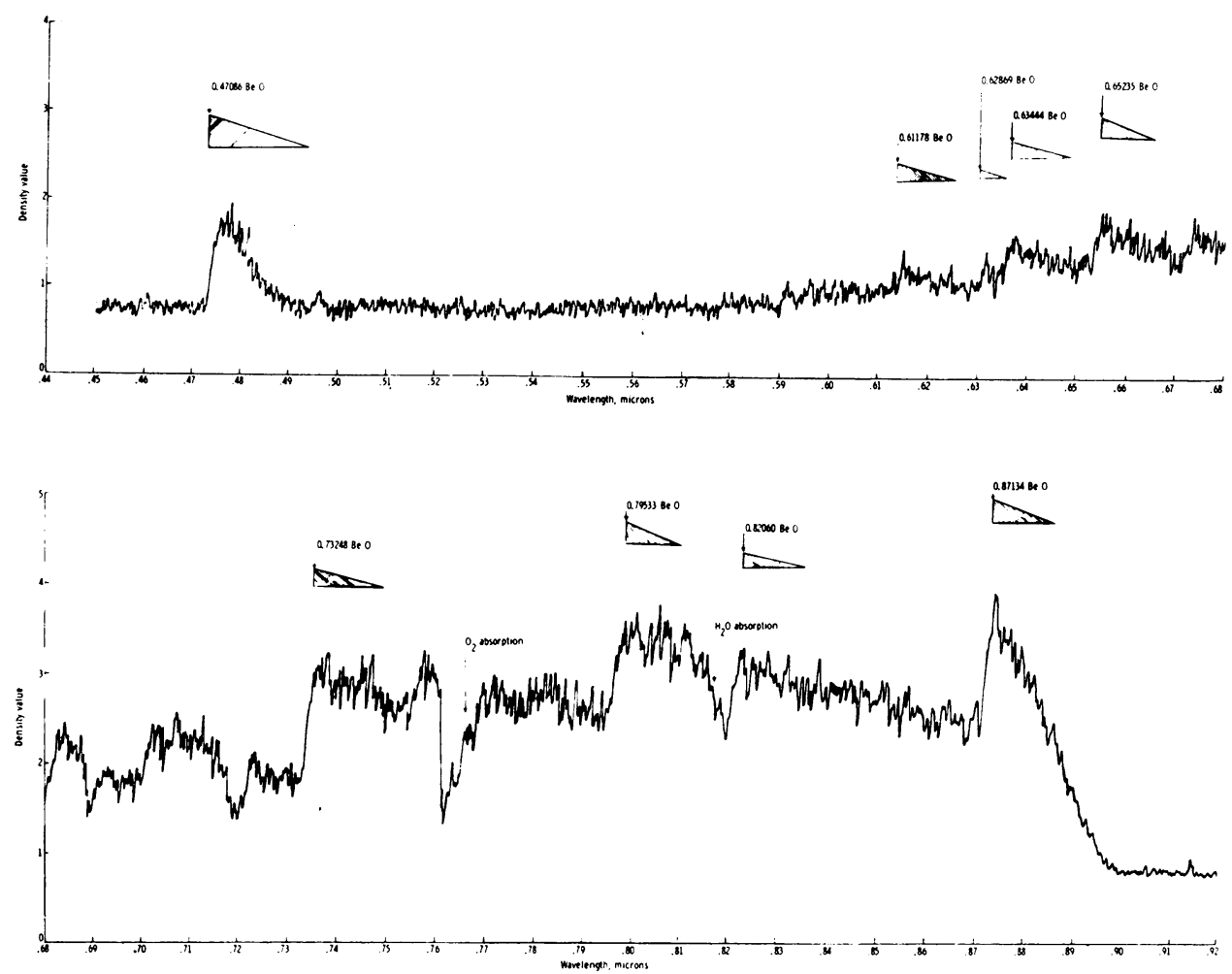

Fig. 6. Density tracing of a spectrum of the Project FIRE re-entry, April 14, 1964, taken from NASA-TN D-3571, 1966. (Courtesy C.W. McKee.)

Table 1

Mean upper-level excitation (volts) of the multiplets of neutral atoms

(Figures in parentheses are the numbers of multiplets averaged)

Prominent features Other features

$\begin{array}{llr}\text { Aurora } & 7.9(3) & 13 \cdot 2(22) \\ \text { Meteors } & 5 \cdot 3(21) & 5 \cdot 7(252) \\ \text { Re-entry Vehicles } & 2 \cdot 8(11) & 5 \cdot 3(190) \\ \text { Rocket Bodies } & 2.5(6) & 4.7(119)\end{array}$

position of the source directly from an identification of the atoms and molecules contributing to the luminosity. The conditions for the appearance of any given element are governed by a large number of factors inherent in the observing conditions, the instrument used, and the physical properties of the atom itself. 


\section{Acknowledgements}

In conclusion, I would like to acknowledge the assistance of a large number of my co-workers who have contributed observational material in advance of publication, in particular, Charles C. Petty and Richard M. Carbone of the Avco Everett Research Laboratory, U.S.A., Zdeněk Ceplecha of the Ondřejov Observatory, Czechoslovakia, and Ian Halliday of the Dominion Observatory, Canada.

\section{References}

Bates, D.R. (1960) Physics of the Upper Atmosphere, ed. by J.A. Ratcliffe, Academic Press, New York, pp. 219-353.

Chamberlain, J.W. (1961) Physics of the Aurora and Airglow, Academic Press, New York.

Gatterer, A., Junkes, J., Salpeter, E. W., Rosen, B. (1957) Molecular Spectra of Metallic Oxides, Specola Vaticana, Vatican City.

McKee, C.W. (1966) NASA Technical Note D-3571.

Millman, P.M. (1963) Smithson. Contr. Astrophys., 7, 119.

Moore, C.E. (1945) Contr. Princeton Obs., 20.

Pearse, R.W.B., Gaydon, A.G. (1963) The Identification of Molecular Spectra, 3rd ed., Chapman and Hall, London. 